Original Article

\title{
Prevention of Atrial Fibrillation with Ultra-Low Dose Landiolol after Off-Pump Coronary Artery Bypass Grafting
}

\author{
Eiki Nagaoka, MD, Hirokuni Arai, MD, PhD, Kiyoshi Tamura, MD, PhD, \\ Satoru Makita, MD, and Naoto Miyagi, MD, PhD
}

\begin{abstract}
Purpose: Postoperative atrial fibrillation (AF) is a common complication of cardiac surgery that is associated with an increased incidence of other complications. The goal of this prospective randomized study was to evaluate the effect of ultra-low dose landiolol hydrochloride for prevention of AF after off-pump coronary artery bypass grafting (CABG). Methods: The subjects were 47 patients who underwent isolated CABG and were randomly divided into those who received landiolol from ICU admission until the beginning of oral drug intake (Group L) and those administered diltiazem hydrochloride over the same period (Group D). The incidence of AF within one week after surgery was examined as the primary endpoint. Heart rate, blood pressure, cardiac output, and other hemodynamic parameters were used as secondary endpoints. The rates of adverse events were also recorded.

Results: The incidences of AF in the first postoperative week were $4.8 \%$ and $27 \%$ in Groups $L$ and $D$, respectively $(p=0.046)$. There were no differences in hemodynamic parameters between the Groups. In multivariate analysis, no factor emerged as a significant risk factor for postoperative AF. Two patients had adverse events of asthma and hypotension, respectively, in Group $\mathrm{L}$.

Conclusion: Ultra-low dose landiolol is effective for preventing AF after CABG without worsening hemodynamics.
\end{abstract}

Keywords: arrhythmia therapy, atrial fibrillation, cardiac function, coronary artery bypass graft, postoperative care

\section{Introduction}

Atrial fibrillation (AF) after cardiac surgery is a common complication that may cause embolism or stroke.

Department of Cardiovascular Surgery, Tokyo Medical and Dental University, Tokyo, Japan

Received: April 9, 2012; Accepted: October 2, 2012

Corresponding author: Hirokuni Arai, MD, PhD. Department of Cardiovascular Surgery, Tokyo Medical and Dental University, 1-5-45 Yushima, Bunkyo-ku, Tokyo 113-8519, Japan

Email: hiro.cvsg@tmd.ac.jp

(C)2014 The Editorial Committee of Annals of Thoracic and Cardiovascular Surgery. All rights reserved.
Therefore, prevention of AF is an important medical and economic issue. ${ }^{1,2)}$ Guidelines $^{3-5)}$ strongly recommend treatment with a beta blocker to prevent postoperative $\mathrm{AF}$ in patients undergoing cardiac surgery. However, an oral beta blocker may cause an unexpected drop in heart rate or blood pressure, and this is a practical reason for not using this kind of drug just after cardiac surgery.

Landiolol hydrochloride is an ultra-short acting $\beta 1$ selective blocker that can be given intravenously and has a half-life of only four minutes. These properties make it easy to titrate the dose, while the high selectivity of landiolol for the $\beta 1$ receptor over the $\beta 2$ receptor (245 times) greatly reduces the chance of adverse events related to bronchial smooth muscle contraction. Thus, 


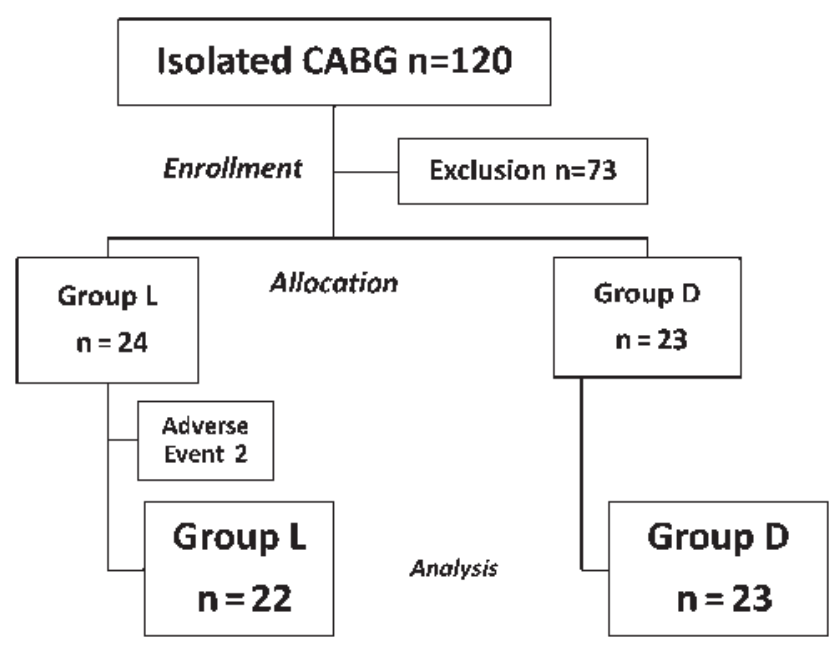

Fig. 1 Disposition of patients who underwent isolated CABG. After exclusion of 73 patients based on the criteria for the study, the remaining 47 patients were randomly assigned to each group. Two patients in Group L were subsequently excluded due to adverse events. CABG: coronary artery bypass grafting.

landiolol may be useful for preventing AF after cardiac surgery. In this study, the effect of ultra-low dose landiolol was examined in a relatively homogeneous population of patients after coronary artery bypass grafting (CABG) to control for the background of the patients and the operative invasiveness.

\section{Methods}

\section{Patients}

Between August 2008 and August 2010, 120 isolated CABG procedures were performed at Tokyo Medical and Dental University Hospital. Of these cases, 47 patients were enrolled in this study (Fig. 1). The exclusion criteria were an emergency operation, preoperative $\mathrm{AF}$, heart rate (HR) $<60 \mathrm{bpm}$, blood pressure $<80 \mathrm{mmHg}$ at the time of admission to the ICU, or patients who did not give consent.

\section{Study design}

This trial was approved by the ethical committee of Tokyo Medical and Dental University. All patients provided written informed consent. After informed consent was obtained, preoperative drug use and medical history were rechecked. If a patient had any history of contraindication to beta blockers, asthma or advanced atrioventricular block, they were excluded from the study.

Patients were randomly assigned to Group L or D using a computer-generated table. In Group L, patients received landiolol from ICU admission until the beginning of oral drug intake. In Group D, patients were administered diltiazem over the same period. Oral drugs were not regulated in either group and were chosen on an individual basis. Landiolol or diltiazem was given under single-blinded conditions from a perspective of safety. The dosage of landiolol was restricted to 0.5 to $2 \mu \mathrm{g} / \mathrm{min} / \mathrm{kg}$ to allow evaluation of the efficacy of landiolol at an ultra-low dose. The dose was varied within this prescribed range based on the judgment of the primary doctor.

\section{Endpoints}

The primary endpoint was the incidence of AF within one week after surgery, based on continuous electrocardiogram (ECG) monitoring over this period. Occurrence of $\mathrm{AF}$ was defined as postoperative new-onset atrial fibrillation or atrial flutter requiring treatment or lasting longer than 5 minutes. The secondary endpoints were hemodynamic data, including HR, systolic blood pressure (SBP) obtained from an arterial line, cardiac index (CI), systolic pulmonary artery pressure (SPA), pulmonary capillary artery wedge pressure (PCWP), and stroke volume index (SVI) obtained from a pulmonary artery catheter. These data were obtained at the time of ICU admission (before drug administration) and at $6 \mathrm{~h}$ after ICU admission (after the start of drug administration). The timing of data collection at $6 \mathrm{~h}$ after ICU admission was chosen to ensure that hemodynamics had stabilized after the start of drug administration. In-hospital mortality and adverse events related to drug administration were recorded until discharge from hospital. If adverse events occurred that were related to landiolol or diltiazem, the drug was stopped and the AF incidence, hemodynamics and other data for the patient were excluded from analysis.

\section{Statistical analysis}

Categorical variables were compared by chi-square test and continuous variables (presented as means \pm SD) were compared by Student t-test and Fisher exact test between Groups L and D. Risk factors for postoperative $\mathrm{AF}$ were compared between patients with and without $\mathrm{AF}$ using univariate analysis of preoperative characteristics, operative values, administered drug (landiolol or diltiazem), and postoperative data. A multivariate analysis was then performed using factors that showed a significant difference in the univariate analysis. 
Table 1 Patient Characteristics

\begin{tabular}{|c|c|c|c|}
\hline preoperative Value & $\begin{array}{c}\text { Group L } \\
n=22\end{array}$ & $\begin{array}{c}\text { Group D } \\
\mathrm{n}=23\end{array}$ & $\mathrm{p}$ Value \\
\hline Age & $67 \pm 8.5$ & $69 \pm 6.3$ & 0.271 \\
\hline Gender (Male) & $91 \%$ & $74 \%$ & 0.074 \\
\hline Hypertension & $68 \%$ & $83 \%$ & 0.351 \\
\hline Hyperlipidemia & $45 \%$ & $74 \%$ & 0.100 \\
\hline Diabetes (Insulin dependent) & $18 \%$ & $23 \%$ & 0.394 \\
\hline Renal failure $(\mathrm{Cr}>2.0 \mathrm{mg} / \mathrm{dL}$ or dialysis $)$ & $9.1 \%$ & $8.7 \%$ & 0.963 \\
\hline Hemodialysis & $4.5 \%$ & $4.3 \%$ & 0.974 \\
\hline Cerebrovascular disease & $14 \%$ & $13 \%$ & 0.953 \\
\hline Smoking & $4.5 \%$ & $13 \%$ & 0.317 \\
\hline $\mathrm{BNP}$ & $72 \pm 91$ & $186 \pm 290$ & 0.086 \\
\hline HR & $64 \pm 10$ & $71 \pm 15$ & 0.098 \\
\hline BMI & $24.3 \pm 2.9$ & $24.2 \pm 2.6$ & 0.916 \\
\hline NYHA class & $1.8 \pm 0.8$ & $2.0 \pm 0.9$ & 0.563 \\
\hline Unstable angina & $23 \%$ & $30 \%$ & 0.800 \\
\hline Urgent or Emergency & $0 \%$ & $0 \%$ & \\
\hline $\operatorname{LVEF}(\%)$ & $64 \pm 12$ & $59 \pm 13$ & 0.220 \\
\hline ACEI & $23 \%$ & $13 \%$ & 0.180 \\
\hline ARBs & $45 \%$ & $47 \%$ & 0.760 \\
\hline$\beta$-blocker & $36 \%$ & $43 \%$ & 0.490 \\
\hline Statin & $50 \%$ & $56 \%$ & 0.520 \\
\hline \multicolumn{4}{|l|}{ Operative Factors } \\
\hline Off pump & $100 \%$ & $100 \%$ & \\
\hline Duration of operation $(\min ) \mathrm{w}$ & $431 \pm 98$ & $448 \pm 110$ & 0.550 \\
\hline RCA revascularization & $77 \%$ & $87 \%$ & 0.180 \\
\hline Number of anastomosis & $3.6 \pm 1.2$ & $4.2 \pm 1.4$ & 0.140 \\
\hline Blood transfusion & $36 \%$ & $52 \%$ & 0.140 \\
\hline
\end{tabular}

\section{Results}

\section{Patients}

There was no difference in preoperative characteristics or operative data between the groups (Table 1). All patients underwent off-pump CABG. The mean numbers of distal anastomoses were $3.6 \pm 1.2$ in Group L and $4.2 \pm 1.4$ in Group D, with no significant difference between the groups. The maximum drug dosage was $0.59 \pm 0.33 \mu \mathrm{g} / \mathrm{kg} / \mathrm{min}$ in Group L and $0.57 \pm 0.31 \mu \mathrm{g} / \mathrm{kg} / \mathrm{min}$ in Group D. Each drug was started at $0.5 \mu \mathrm{g} / \mathrm{kg} / \mathrm{min}$ and maintained at the same dosage in all except three patients (two in Group L and one in Group D) who received a dose in the range of 0.5 to $2.0 \mu \mathrm{g} / \mathrm{kg} / \mathrm{min}$. The mean dosing periods were $1.6 \pm 0.7$ days in Group $L$ and $2.4 \pm 1.7$ days in Group D, with no significant difference between the groups $(p=0.054)$. Two patients in Group L were eliminated from the analysis because landiolol had to be stopped due to adverse effects of asthma and hypotension, respectively.

\section{Endpoints}

The incidence of AF in the week after surgery was significantly lower in Group L than in Group D [1/21 (4.8\%) vs. $6 / 22(27 \%), p=0.046]$. The average AF onset day was $4.4 \pm 2.2$ postoperative days ( 3 in Group $\mathrm{L}$ and $4.7 \pm 2.3$ in Group D). Hemodynamic parameters did not differ significantly between the groups (Fig. 2). However, SBP decreased in both groups, and SPA and PCWP showed significant decreases in Group L $(p<0.05)$. The incidence of adverse events was $2(8.3 \%)$ in Group L: one case each of asthma and hypotension. For these two patients, landiolol was stopped immediately after the beginning of administration. There were no adverse events in Group D and there were no hospital deaths in either group. 

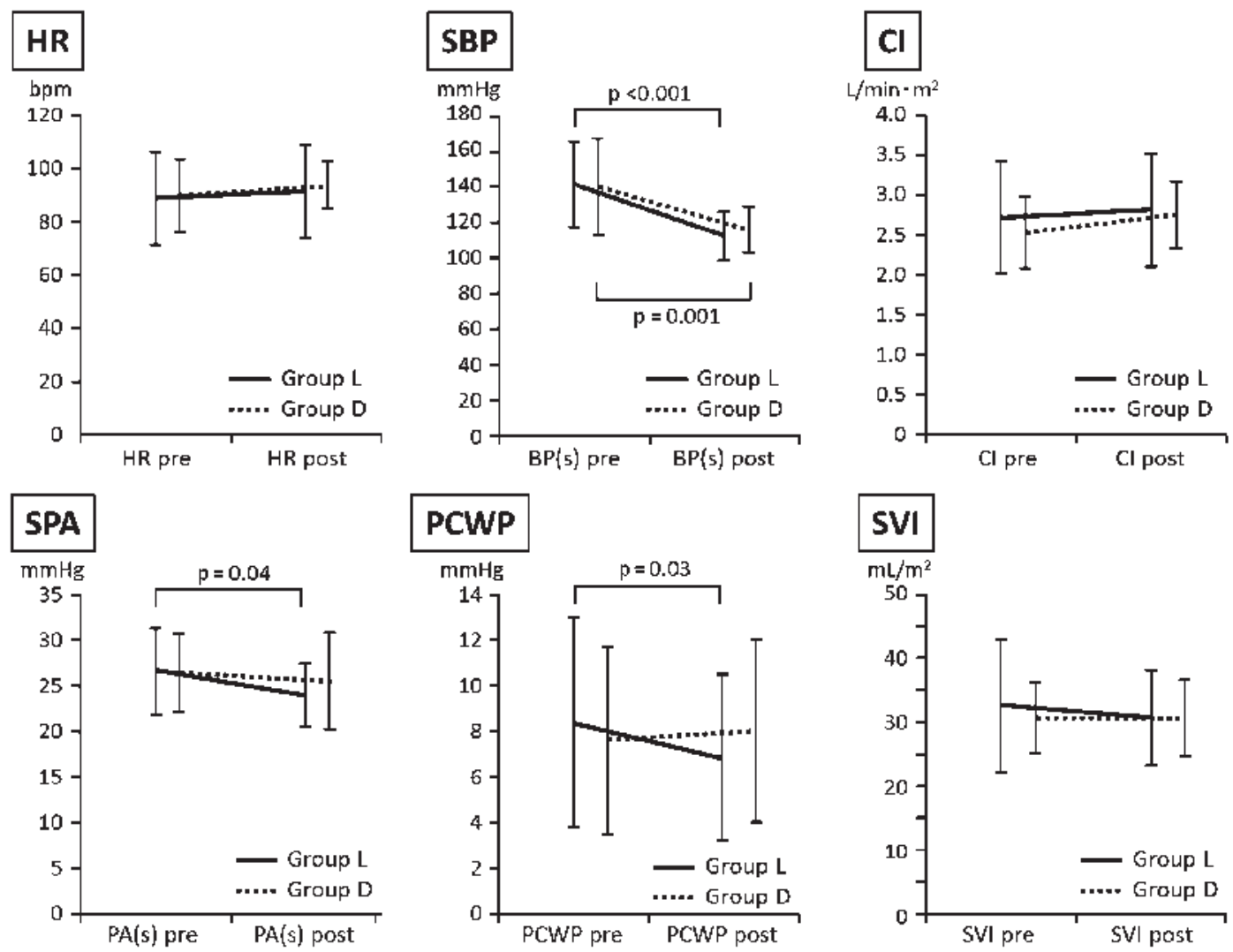

Fig. 2 Hemodynamic changes in heart rate (HR), systolic blood pressure (SBP), systolic pulmonary artery pressure (SPA), cardiac index (CI), pulmonary capillary wedge pressure (PCWP), and stroke volume index (SVI) from pre- to post-drug administration.

\section{Risk factors for postoperative atrial fibrillation}

Preoperative factors, operative values, administered drug, and postoperative factors were compared between patients with $\mathrm{AF}(\mathrm{n}=7)$ and without $\mathrm{AF}(\mathrm{n}=38)$ in logistic regression analysis (Table 2). In univariate analysis, there was a propensity for differences in non-use of landiolol (Group D), preoperative left atrium dimension, preoperative NYHA class, body mass index (BMI), and $\mathrm{CI}$ before drug administration between patients with and without AF. In multivariate analysis, no factor emerged as a significant risk factor for postoperative $\mathrm{AF}(\mathrm{p}<0.05)$. However, non-use of landiolol (OR $=0.04$ [95\% Confidence interval $=0.00-1.08], \mathrm{p}=0.0554$ ), preoperative left atrium dimension $(\mathrm{OR}=1.48[95 \%$ Confidence interval $=0.96-2.24], \mathrm{p}=0.0652)$, and CI before drug administration $(\mathrm{OR}=0.06$ [95\% Confidence interval $=$ $0.00-1.63], \mathrm{p}=0.0963$ ) had a propensity to be risk factors $(\mathrm{p}<0.1)$.

\section{Discussion}

In this study, we showed that ultra-low dose landiolol administration after off-pump CABG reduced the incidence of postoperative new onset AF. The utility of landiolol for preventing postoperative $\mathrm{AF}$ has been reported widely, ${ }^{6-8)}$ but this is the first prospective randomized trial of postoperative landiolol and the dose $(0.5$ to $2 \mu \mathrm{g} / \mathrm{kg} / \mathrm{min})$ is the lowest for postoperative prophylactic administration. The ideal approach is to prevent postoperative AF without worsening hemodynamics, and this was achieved with ultra-low dose landiolol administration in this study. In fact, SPA and PCWP decreased, indicating probable improvement of hemodynamics.

Diltiazem, a nondihydropyridine calcium channel antagonist, lengthens refractoriness and slows conduction across the AV node. In the absence of preexcitation, intravenous administration of diltiazem is recommended to slow the ventricular response to AF. ${ }^{9)}$ However, there is no evidence to support antiarrhythmic efficacy of diltiazem in patients with paroxysmal AF, and also no evidence of a propensity to promote AF with diltiazem. In this study, the efficacy of landiolol for preventing AF was shown in comparison to diltiazem. Given the lack of effects of diltiazem on AF, this result is essentially 
Table 2 Risk factors for postoperative atrial fibrillation

\begin{tabular}{|c|c|c|c|c|}
\hline & \multicolumn{2}{|l|}{ Univariate Analysis } & \multicolumn{2}{|l|}{ Multivariate Analysis } \\
\hline & OR [95\% Confidence interval] & $\mathrm{p}$ Value & OR [95\% Confidence interval] & $\mathrm{p}$ Value \\
\hline \multicolumn{5}{|l|}{ Preoperative factors } \\
\hline Sex (male) & $0.47[0.07-3.01]$ & 0.424 & & \\
\hline Age & $1.09[0.94-1.26]$ & 0.246 & & \\
\hline Hypertension & $3.21[0.58-17.74]$ & 0.180 & & \\
\hline Hyperlipidemia & $4.57[0.50-41.88]$ & 0.179 & & \\
\hline Diabetes (Insulin dependent) & $2.02[0.35-11.76]$ & 0.432 & & \\
\hline BNP & $1.00[1.00-1.01]$ & 0.071 & & \\
\hline HR & $1.02[0.95-1.10]$ & 0.520 & & \\
\hline BMI & $1.33[0.90-1.97]$ & 0.142 & $0.62[0.35-1.12]$ & 0.1136 \\
\hline NYHA & $2.52[0.72-8.82]$ & 0.147 & $0.22[0.03-1.44]$ & 0.1136 \\
\hline Beta blocker & $1.23[0.20-7.70]$ & 0.827 & & \\
\hline Statin & $2.19[0.43-11.27]$ & 0.348 & & \\
\hline ACEI & $2.13[0.33-13.67]$ & 0.783 & & \\
\hline $\mathrm{ARBs}$ & $0.40[0.07-2.32]$ & 0.527 & & \\
\hline \multicolumn{5}{|l|}{ Echocardiographic data } \\
\hline LVDd & $1.08[0.94-1.23]$ & 0.264 & & \\
\hline LAD & $1.13[0.97-1.32]$ & 0.117 & $1.48[0.96-2.24]$ & 0.0652 \\
\hline LVEF & $1.04[0.98-1.10]$ & 0.227 & & \\
\hline \multicolumn{5}{|l|}{ Operative values } \\
\hline Duration of operation & $1.00[0.99-1.01]$ & 0.541 & & \\
\hline Bypass number & {$[-]$} & & & \\
\hline RCA revascularization & $2.13[0.33-13.67]$ & 0.424 & & \\
\hline \multicolumn{5}{|l|}{ Postoperative factors } \\
\hline Group (D=0, L = 1) & $7.41[0.81-67.62]$ & 0.076 & 0.04 [0.00-1.08] & 0.0554 \\
\hline HR pre & $0.95[0.88-1.01]$ & 0.102 & & \\
\hline SBP pre & $0.98[0.95-1.02]$ & 0.302 & & \\
\hline CI pre & 6.87 [0.84-55.94] & 0.072 & $0.06[0.00-1.63]$ & 0.0963 \\
\hline CRP (OPOD) & $3.78[0.78-18.40]$ & 0.099 & & \\
\hline $\operatorname{maxCKMB}$ & $1.02[0.98-1.06]$ & 0.257 & & \\
\hline
\end{tabular}

OR: odds ratio; BNP: brain natriuretic peptide; HR: heart rate on admission; BMI: body mass index; NYHA: New York Heart Association; ACEI: angiotensin converting enzyme inhibitor; ARBs: angiotensin receptor blockers; LVDd: left ventricular end-diastolic dimension; LAD: left atrium dimension; LVEF: left ventricle ejection fraction; RCA: right coronary artery; SBP: systolic blood pressure; pre: before drug administration; $\mathrm{CI}$ : cardiac index; CRP (OPOD): C reactive protein at 0 postoperative day; CKMB: creatine phosphokinase MB isoenzyme

equivalent to a placebo-controlled study.

There are two results in the study that are difficult to explain. First, the common mechanism of prevention of AF with a beta blocker is thought to occur via an antiischemic effect through decreased cardiac oxygen consumption. However, we found no evidence of hemodynamic changes with landiolol use. Thus, the mechanism of prevention of AF with ultra-low dose landiolol appears not to be related to cardiac oxygen consumption.

A second unusual result was that the duration of $\mathrm{AF}$ prevention and that of drug administration were substantially different. As mentioned above, landiolol is a short-acting beta blocker with a short half-life. However, $\mathrm{AF}$ was reduced for 7 days with administration of landiolol for only $1.5 \pm 0.7$ days, indicating prolongation of the pharmacological effect. There are many studies in which beta blockers have been associated with myocardial protection through anti-ischemic, anti-inflammatory and sympathoinhibitory effects. ${ }^{8,10)}$ With continuous intravenous beta blocker administration, the circadian variability of heart rate or blood pressure would be inhibited more than with oral beta blocker intake. For example, Hirata, et al. reported the utility of low-dose $(30 \mu \mathrm{g} / \mathrm{kg} /$ min, higher than in the current study) continuous landiolol infusion for reducing the adrenergic response during tracheal intubation in elderly patients. ${ }^{11)}$ Landiolol inhibited an increase in heart rate and systolic blood pressure after intubation compared with a control group, whereas these values did not differ before tracheal intubation. In this trial, the variability was not recorded, but the 
sympathoinhibitory effect might have decreased total cardiac oxygen consumption during landiolol administration and the anti-ischemic effect may have been prolonged after landiolol was stopped.

Anti-inflammatory effects of beta blockers and their involvement in $\mathrm{AF}$ prevention have also been reported, ${ }^{8,12)}$ but we were unable to identify an anti-inflammatory effect of ultra-low dose landiolol based on postoperative CRP levels at $0,1,2$ and 7 postoperative days, which did not differ between the groups or between patients with and without AF. Further evaluation of IL-6, IL-8, high sensitivity CRP and other chemical mediators may be required to detect an anti-inflammatory effect of landiolol.

Our study has some limitations. First, the trial was performed under single-blinded conditions from a consideration of safety. Based on the effect of landiolol or diltiazem, the primary physician could change the amount of inotropic agents or choose other therapeutic options. In principle, when a drug effect is investigated clinically, it should be compared with placebo under double-blinded conditions with a fixed period of administration. However, in this trial, landiolol was compared with diltiazem, rather than a placebo, because diltiazem is routinely used for preventing AF and vasospasm in our hospital. A second limitation was the relatively small sample size, and a larger study is needed for further clarification of the effect of landiolol on prevention of AF.

\section{Conclusion}

Within the limitations stated above, we conclude that ultra-low dose landiolol administration is effective for preventing $\mathrm{AF}$ after $\mathrm{CABG}$ without causing worsening of hemodynamics.

\section{Disclosure Statement}

None of the authors have a conflict of interest regarding the work in the study.

\section{References}

1) Mariscalco G, Klersy C, Zanobini M, et al. Atrial fibrillation after isolated coronary surgery affects late survival. Circulation 2008; 118: 1612-8.

2) Ngaage DL, Schaff HV, Mullany CJ, et al. Does preoperative atrial fibrillation influence early and late outcomes of coronary artery bypass grafting? J Thorac Cardiovasc Surg 2007; 133: 182-9.
3) Martinez EA, Bass EB, Zimetbaum P, et al. Pharmacologic control of rhythm: American College of Chest Physicians guidelines for the prevention and management of postoperative atrial fibrillation after cardiac surgery. Chest 2005; 128(2 Suppl): 48S-55S.

4) Fuster V, Rydén LE, Cannom DS, et al. 2011 ACCF/ AHA/HRS focused updates incorporated into the ACC/AHA/ESC 2006 Guidelines for the management of patients with atrial fibrillation: a report of the American College of Cardiology Foundation/American Heart Association Task Force on Practice Guidelines developed in partnership with the European Society of Cardiology and in collaboration with the European Heart Rhythm Association and the Heart Rhythm Society. J Am Coll Cardiol 2011; 57: e101-98.

5) Task Force on Myocardial Revascularization of the European Society of Cardiology (ESC) and the European Association for Cardio-Thoracic Surgery (EACTS); European Association for Percutaneous Cardiovascular Interventions (EAPCI), Kolh P, et al. Guidelines on myocardial revascularization. Eur J Cardiothorac Surg 2010; 38(Suppl): S1-S52.

6) Fujiwara H, Sakurai M, Namai A, et al. Effect of lowdose landiolol, an ultrashort-acting beta-blocker, on postoperative atrial fibrillation after CABG surgery. Gen Thorac Cardiovasc Surg 2009; 57: 132-7.

7) Yoshioka I, Sakurai M, Namai A, et al. Postoperative treatment of carvedilol following low dose landiolol has preventive effect for atrial fibrillation after coronary artery bypass grafting. Thorac Cardiovasc Surg 2009; 57: 464-7.

8) Sezai A, Minami K, Nakai T, et al. Landiolol hydrochloride for prevention of atrial fibrillation after coronary artery bypass grafting: new evidence from the PASCAL trial. J Thorac Cardiovasc Surg 2011; 141: 1478-87.

9) Fuster V, Rydén LE, Cannom DS, et al. 2011 ACCF/ AHA/HRS focused updates incorporated into the ACC/AHA/ESC 2006 guidelines for the management of patients with atrial fibrillation: a report of the American College of Cardiology Foundation/American Heart Association Task Force on practice guidelines. Circulation 2011; 123: e269-367.

10) Stevens RD, Burri H, Tramèr MR. Pharmacologic myocardial protection in patients undergoing noncardiac surgery: a quantitative systematic review. Anesth Analg 2003; 97: 623-33.

11) Hirata N, Miyashita R, Watanabe A, et al. Low-dose continuous infusion of landiolol can reduce adrenergic response during tracheal intubation in elderly patients with cardiovascular disease. J Anesth 2010; 24: 786-8.

12) Doo YC, Kim DM, Oh DJ, et al. Effect of beta blockers on expression of interleukin-6 and C-reactive protein in patients with unstable angina pectoris. Am J Cardiol 2001; 88: 422-4. 